\title{
RELAXED ELASTIC LINE ON A CURVED SURFACE*
}

\author{
BY \\ GERALD S. MANNING \\ Rutgers, The State University of New Jersey
}

\begin{abstract}
In an effort to begin to understand the mechanics of various forms of biologically packaged DNA, we develop the Euler-Lagrange equations for the equilibrium path of an elastic line constrained to a surface but otherwise relaxed. We find, in contrast to a statement by Hilbert and Cohn-Vossen [1], that whether or not the solutions are geodesic curves of the surface depends on the boundary conditions and on the surface. Not surprisingly, the relaxed elastic line on a plane or a sphere is always a geodesic (straight line and great circle, respectively). On a cylinder and a "pseudotorus," however, the relaxed line is a geodesic only if both ends are free. For example, a relaxed line on a cylinder, with fixed initial point and oblique tangent, does not wind on the corresponding geodesic (helix).
\end{abstract}

1. Introduction. An elastic line of length $l$ is defined as a curve with associated energy

$$
K=\int_{0}^{l} \kappa^{2}(s) d s
$$

where $s$ is the arc length along the curve and $\kappa^{2}(s)$ is the square curvature. The integral $K$ is called the total square curvature. If a thin rod of invariant length $l$ and uniform circular cross section has a bending stiffness constant equal to two, and if no twisting moments are applied at its ends (so that the torsional deformation vanishes everywhere along the rod [2]), then $K$ is the Hooke's Law elastic energy of the rod. The results of this paper are independent of the value of the Hooke's Law stiffness constant.

If no boundary conditions are imposed at $s=l$, and if no external forces act at any $s$, the elastic line is relaxed. The trajectory of a relaxed elastic line in space or on a plane is a straight line because the positive indefinite quantity that defines $K$ takes its minimum value of zero when the square curvature vanishes for all $s$. The trajectory of a relaxed elastic line constrained to lie on a general surface is, however, dependent on the intrinsic curvature of the surface, which in general bounds the possible values of $K$ away from zero.

* Received July 15, 1986.

This research has been partially supported by NIH Grant GM 36284-01

(c) 1987 Brown University 
Hilbert and Cohn-Vossen [1] (p. 221) state incorrectly that a relaxed elastic line with specified position and tangent at $s=0$ always has the trajectory of a geodesic (the analog for a general surface of the straight line in a plane). We discuss some restrictive conditions for the validity of their statement, but we also demonstrate that a relaxed elastic line on a circular cylinder with initial tangent oblique to a generator does not follow a helical path (the geodesics of a cylinder are the helices), except in the limit $l \rightarrow 0$, nor does an elastic line on a torus conform to the geodesics of the torus.

Physical motivation for study of the problem of elastic lines on surfaces may be found in the nucleosome core particle [3, 4]. In this biological structure the long axis of a stiff segment of DNA is wound roughly as a helix around a roughly cylindrical protein surface (histone octamer). We wondered whether the trajectory of the DNA could be understood as determined only by the action of uniform surface attraction. Is DNA helically wound on the outside of the histone octamer because a relaxed elastic line (model for DNA) assumes the form of a helix when confined to a cylinder? The answer is no.

A more complete question, of course, would be whether the observed helical path can be explained as the outcome of minimization of the positive bending energy coupled to maximization of the negative energy of attraction to the surface. An infinite cylinder is then not an adequate model; the nucleosome-solvent boundary, that is, the ends of the cylinder, will have to be considered. We do not attempt to explore this more difficult problem here.

Polynucleosomes, which are perhaps the fundamental fibrous unit of chromosomes, pack into a shallow superhelical structure [3]. One turn of the superhelix resembles a torus (protein) with a DNA polymer wound around it [5]. Moreover, DNA with neutralized charge self-aggregates into a torus-shaped particle [6]. The stability of a DNA torus, and certainly the dynamics of its formation, may depend on the winding of surface strands of DNA. We are therefore interested in the mechanics of an elastic line on a torus as well as on a cylinder.

The DNA molecule itself looks remarkably like a helicoid. Perhaps the adsorption of polylysine or polyamines to DNA can be modeled as an elastic line constrained to a helicoidal surface, but the analysis of an elastic line on a helicoid will be reserved for subsequent analysis.

To approach the problem, we develop a general apparatus which uses only well-known facts, definitions, and manipulations from differential geometry [7] and the calculus of variations [8]. As an illustration, we apply the general formalism to a plane, where the results are known beforehand. The analysis of a sphere will produce no surprises; the relaxed elastic line does always lie along a geodesic, a great circle. The geodesics of a cylinder and a torus provide solutions for the relaxed elastic line only in special cases, however.

In a subsequent paper we will present affirmative results for an elastic line relaxed on a cylinder. The line winds around the cylinder but not as a helix. The onset of elastic instability at a critical length can also be demonstrated. There is a precise sense in which an elastic line adsorbed on a cylinder can be regarded as more flexible than the unconstrained line, and the analysis of flexibility will be the subject of a subsequent 
publication as well. Ultimately, we hope to understand the mechanics of nucleosomal DNA, but our experience thus far suggests that only a thorough analysis, starting with the simplest models, will suffice.

2. The differential elastic line. The conclusion of Hilbert and Cohn-Vossen [1] that a relaxed elastic line always follows a geodesic is incorrect, but only because the authors indulge in an unwarranted descriptive extrapolation from an exact result of differential geometry. In the differential sense, that is, as the length $l$ of the elastic line tends to zero, their statement is accurate.

Consider a point $\mathbf{x}_{0}$ on a surface $S$ and a unit surface tangent vector $\mathbf{t}_{0}$ at $\mathbf{x}_{0}$. Let $F\left(\mathbf{x}_{0} ; \mathbf{t}_{0}\right)$ be the family of curves on $S$ passing through $\mathbf{x}_{0}$ with direction $\mathbf{t}_{0}$. In this family of curves some have principal normal vectors at $\mathbf{x}_{0}$ that coincide with the unit surface normal vector of $S$ at $\mathbf{x}_{0}$. These curves are placed in the subfamily $F_{1}$, while all the other curves in $F$ form subfamily $F_{2}$. Let $\theta_{0}$ be the angle, for any curve $C$ in $F$, between the principal normal vector of $C$ at $\mathbf{x}_{0}$ and the surface normal at $\mathbf{x}_{0}$. Curves in $F_{1}$ have $\theta_{0}=0$, hence share a common osculating plane (the plane determined for each point on a curve by the tangent and principal normal at that point).

There are relations among $\theta_{0}$, the curvature $\kappa_{0}$ of $C$ at $\mathbf{x}_{0}$, the normal curvature $\kappa_{N}^{0}$ of the surface $S$ at $\mathbf{x}_{0}$ in the direction $\mathbf{t}_{0}$, and the geodesic curvature $\kappa_{g}^{0}$ of $C$ at $\mathbf{x}_{0}$,

$$
\begin{aligned}
& \kappa_{N}^{0}=\kappa_{0} \cos \theta_{0}, \\
& \kappa_{g}^{0}=\kappa_{0} \sin \theta_{0}, \\
& \kappa_{0}^{2}=\kappa_{N}^{0^{2}}+\kappa_{g}^{0^{2}} .
\end{aligned}
$$

The normal curvature $\kappa_{N}^{0}$ is the curvature of the normal section at $\mathbf{x}_{0}$ in direction $\mathbf{t}_{0}$. The normal section is the plane curve of intersection of $S$ with the plane determined by $\mathbf{t}_{0}$ and the surface normal at $\mathbf{x}_{0}$; the normal section is one of the member curves of $F$. The geodesic curvature $\kappa_{g}^{0}$ of $C$ at $\mathbf{x}_{0}$ is the curvature at $\mathbf{x}_{0}$ of the orthogonal projection of $C$ onto the plane tangent to $S$ at $\mathbf{x}_{0}$.

Those curves $C$ in $F$ that are also in $F_{1}$ are characterized by $\theta_{0}=0$. Equation (3) states that their geodesic curvatures vanish at $\mathbf{x}_{0}$. Among the curves in $F_{1}$ are the normal section and the single geodesic in $F$. The normal section is in $F_{1}$ because, as a plane curve, its principal normal at $\mathbf{x}_{0}$ obviously coincides with the surface normal at $\mathbf{x}_{0}$, so $\theta_{0}=0$. The geodesic in $F$ (there is only one because there is a unique geodesic on a surface that passes through a given point with given tangent at that point), like all geodesics, has zero geodesic curvature everywhere, in particular at $\mathbf{x}_{0}$, so $\theta_{0}=0$ from Eq. (3). In addition to the normal section and the geodesic, $F_{1}$ contains an infinite continuum of other curves. All have vanishing geodesic curvature at $\mathbf{x}_{0}$; only the geodesic has identically vanishing geodesic curvature.

We can narrow consideration to the arc of each curve in $F$ that starts at $\mathbf{x}_{0}$ and extends along the curve for a fixed length $l$. Let $F, F_{1}$, and $F_{2}$ now mean the corresponding families of arcs. Because the arcs all have the same length $l$, they share a common arc-length parameter $s, 0 \leqslant s \leqslant l, s=0$ at $\mathbf{x}_{0}$. 
Separately from the differential geometry of curves on a surface, let us regard an elastic line of length $l$ positioned on $S$ with its initial point at $\mathbf{x}_{0}$ and its initial tangent along $\mathbf{t}_{0}$. There is a one-to-one correspondence between the possible trajectories of the elastic line and the arcs of the family $F$. For any of its trajectories, say $a$,

$$
K_{a}=\int_{0}^{l} \kappa_{a}^{2}(s) d s=l \kappa_{a}^{2}\left(\lambda_{a} l\right), \quad 0 \leqslant \lambda_{a} \leqslant 1 .
$$

For any two of its possible trajectories, $a$ and $b$, from Eq. (4),

$$
\begin{aligned}
\lim _{l \rightarrow 0}\left(K_{a} / K_{b}\right) & =\kappa_{a}^{2}\left(\mathbf{x}_{0}\right) / \kappa_{b}^{2}\left(\mathbf{x}_{0}\right) \\
& =\left[\kappa_{N}^{2}\left(\mathbf{x}_{0}\right)+\kappa_{g, a}^{2}\left(\mathbf{x}_{0}\right)\right] /\left[\kappa_{N}^{2}\left(\mathbf{x}_{0}\right)+\kappa_{g, b}^{2}\left(\mathbf{x}_{0}\right)\right] .
\end{aligned}
$$

In this equation it is important to notice that $\kappa_{N}\left(\mathbf{x}_{0}\right)$ is a property of the surface, not of the particular curves $a$ and $b$. Now if $a$ and $b$ are both in $F_{1}$, then $\kappa_{g, a}=\kappa_{g, b}=0$, and $\lim _{l \rightarrow 0}\left(K_{a} / K_{b}\right)=1$. If $a$ is in $F_{1}$, but $b$ is in $F_{2}$, then $\kappa_{g, a}=0$, while $\kappa_{g, b} \neq 0$, so $\lim _{l \rightarrow 0}\left(K_{a} / K_{b}\right)<1$.

We conclude that in the limit of zero length the relaxed (lowest energy) trajectory of the elastic line fluctuates among the arcs of the family $F_{1}$ with equal probability of being along any one of them. Among these arcs is the geodesic starting at $\mathbf{x}_{0}$ in direction $\mathbf{t}_{0}$. Of course, thermal fluctuations carry the elastic line into the arcs of $F_{2}$ also, but these trajectories have higher energy and lower probability.

3. Geodesic as globally relaxed elastic line. The result obtained in the preceding section is of limited physical interest. The pertinent problem is to identify that curve in a family of curves of fixed length $l$ which has least total square curvature [Eq. (1)]. In general the minimizing curve is not a geodesic. It is a geodesic, however, in the circumstances described below.

Let

$$
K_{N}=\int_{0}^{l} \kappa_{N}^{2}(s) d s
$$

be called the total square normal curvature of a curve $C_{l}$ of length $l$. Let $\bar{C}_{l}$ be the curve that minimizes $K_{N}$ among all curves of length $l$ on a surface $S$ with stated boundary conditions at $s=0$ and $s=l$. Properties of $\bar{C}_{l}$ will be designated by a bar; corresponding properties of a general curve $C_{l}$ will not have a bar. For any curve $C_{l}$ on $S$ with the stated boundary conditions, $K=\int_{0}^{l} \kappa^{2} d s=\int_{0}^{l}\left(\kappa_{N}^{2}+\kappa_{g}^{2}\right) d s \geqslant \int_{0}^{l} \kappa_{N}^{2} d s=K_{N} \geqslant \bar{K}_{N}$, while, if $\bar{C}_{l}$ is a geodesic, $\bar{K}=\int_{0}^{l} \bar{\kappa}^{2} d s=\int_{0}^{l}\left(\bar{\kappa}_{N}^{2}+\bar{\kappa}_{g}^{2}\right) d s=\int_{0}^{l} \bar{\kappa}_{N}^{2} d s=\bar{K}_{N}$, since $\bar{\kappa}_{g}$ identically vanishes. Hence $K \geqslant \bar{K}$. In words, if the curve that has least total square normal curvature is a geodesic, then this curve has least total square curvature.

To implement this rule for a given surface, we first must find the curve that minimizes $K_{N}$. Then we must show that this curve is a solution of the differential equations of geodesic curves for the given surface. If it is, then we have proved that the relaxed elastic line follows a geodesic trajectory. 
4. The incomplete variational problem. Let $\mathbf{x}$ be a three-component vector from some fixed origin to a general point on a surface $S$. Let $(u, v)$ be surface coordinates on $S$ so that $\mathbf{x}(u, v)$ is the general point of the surface. A curve $C$ on the surface with arc length $s$ is specified by $[u(s), v(s)]$, and the three-dimensional vector $\mathbf{x}$ with tip tracing out the path of the curve is $\mathbf{x}[u(s), v(s)]$. The unit tangent vector along the curve is $\mathbf{t}=d \mathbf{x} / d s=$ $\mathbf{x}_{u} u^{\prime}+\mathbf{x}_{v} v^{\prime}$, where $\mathbf{x}_{u}$ and $\mathbf{x}_{v}$, are, respectively, the partial derivatives of $\mathbf{x}$ with respect to $u$ and $v$, and the prime means differentiation with respect to $s$. Since $\mathbf{t} \cdot \mathbf{t}=1$, we have a constraining relation between any pair of functions $[u(s), v(s)]$ that define a curve on the surface,

$$
g\left(u, v, u^{\prime}, v^{\prime}\right)=1
$$

where

$$
g=E u^{\prime 2}+2 F u^{\prime} v^{\prime}+G v^{\prime 2}
$$

and $E=\mathbf{x}_{u} \cdot \mathbf{x}_{u}, F=\mathbf{x}_{u} \cdot \mathbf{x}_{v}, G=\mathbf{x}_{v} \cdot \mathbf{x}_{v}$ are the three coefficients of the first fundamental form.

The square normal curvature can be expressed in terms of the coordinates $[u(s), v(s)]$ along the curve,

$$
\kappa_{N}^{2}(s)=\left(L u^{\prime 2}+2 M u^{\prime} v^{\prime}+N v^{\prime 2}\right)^{2},
$$

where $L, M$, and $N$ are the coefficients of the second fundamental form. Formulas are available to express these coefficients in terms of $\mathbf{x}_{u}, \mathbf{x}_{v}$, and the second derivatives $\mathbf{x}_{u u}$, $\mathbf{x}_{u v}, \mathbf{x}_{i,}$; so $L, M$, and $N$ are generally functions of $u$ and $v$ [7]. The problem at hand, then, is to find functions $u(s)$ and $v(s)$ that give stationary values to the integral

$$
K_{N}=\int_{0}^{l} \kappa_{N}^{2}\left(u, v, u^{\prime}, v^{\prime}\right) d s
$$

subject to the side condition Eq. (8). We call this problem incomplete because it seeks to minimize the total square normal curvature, not the total square curvature (total energy), as part of the implementation of the rule in the preceding section.

The Euler equations for the incomplete problem are:

$$
\begin{gathered}
H_{u}-\left(H_{u^{\prime}}\right)^{\prime}=0, \\
H_{v^{\prime}}-\left(H_{v^{\prime}}\right)^{\prime}=0,
\end{gathered}
$$

where

$$
H=\kappa_{N}^{2}+\lambda(g-1)
$$

and $\lambda=\lambda(s)$ is a Lagrange multiplier function. Equations (8), (12), and (13) are a system of three equations to determine the three functions $u(s), v(s)$, and $\lambda(s)$. The system is of fifth order; second order in $u$ and $v$, first order in $\lambda$. There are five constants of integration in the general solution. One of them is fixed by the following argument. Differentiate Eq. (8) once with respect to $s$ to make it second order in $u$ and $v$, so that the fifth-order system of equations is thrown into normal form. When the resulting equation $g^{\prime}=0$ is integrated, Eq. (8) dictates that the constant of integration equal unity. 
We turn now to the boundary conditions at $s=0$ and $s=l$ that determine the other four constants of integration. In the expression for the variation there appear four boundary terms resulting from the usual integration by parts. These terms are $H_{u^{\prime}} \delta u$ and $H_{v}, \delta v$, each evaluated at $s=0$ and at $s=l$. Consider an elastic line free to be pulled by energy-minimizing forces anywhere on the surface, in any direction. For these "natural" boundary conditions at $s=0$ and $s=l$,

$$
H_{u^{\prime}}=H_{r^{\prime}}=0 \quad(s=0, l),
$$

thus determining four constants of integration. If the initial point of the elastic line is fixed, say,

$$
u(0)=u_{0}, \quad v(0)=v_{0},
$$

but the end of the line is free, then

$$
H_{u^{\prime}}=H_{u^{\prime}}=0 \quad(s=l) .
$$

The four integration constants are now determined by Eqs. (16) and (17). Specification of both initial position and direction is incompatible with the incomplete variational problem.

5. The complete variational problem. We will see that the method of the preceding two sections is applicable to the plane, cylinder, and sphere, but fails to discover the relaxed elastic line on a torus. Further, it cannot be used if we want to specify the initial tangent to the elastic line-and it is this problem that turns out to give a nongeodesic curve for the relaxed line. We have no choice but to investigate the complete variational problem, which seeks to minimize the total square curvature, Eq. (1).

We have

$$
\kappa^{2}=\kappa_{N}^{2}+\kappa_{g}^{2},
$$

where $\kappa_{N}^{2}$ is given by Eq. (10). We can work out an expression for $\kappa_{g}^{2}$ in terms of the coordinates $[u(s), v(s)]$ and find

$$
\kappa_{g}^{2}=E \gamma_{1}^{2}+2 F \gamma_{1} \gamma_{2}+G \gamma_{2}^{2},
$$

where

$$
\begin{aligned}
& \gamma_{1}=u^{\prime \prime}+\Gamma_{11}^{1} u^{\prime 2}+2 \Gamma_{12}^{1} u^{\prime} v^{\prime}+\Gamma_{22}^{1} v^{\prime 2}, \\
& \gamma_{2}=v^{\prime \prime}+\Gamma_{11}^{2} u^{\prime 2}+2 \Gamma_{12}^{2} u^{\prime} v^{\prime}+\Gamma_{22}^{2} v^{\prime 2} .
\end{aligned}
$$

The quantities $\Gamma_{i j}^{k}$ are the Christoffel symbols of the second kind; available formulas express them as functions of $E, F$, and $G$, and their first partial derivatives with respect to $u$ and $v$ [7].

We note here that $E, F$, and $G$ are the coefficients of a positive definite quadratic form (the first fundamental form equals $d s^{2}$ ). Hence, the equations of a geodesic curve, which is characterized by identically vanishing $\kappa_{g}$, must be given by $\gamma_{1}=\gamma_{2}=0$, and indeed they are [7]. 
Through $\gamma_{1}$ and $\gamma_{2}, \kappa^{2}$ has a dependence on $u^{\prime \prime}$ and $v^{\prime \prime}$ in addition to $u, v, u^{\prime}$, and $v^{\prime}$. The Euler equations which yield functions $u(s)$ and $v(s)$ that are stationary for the integral $K$ of Eq. (1) are of order higher than before,

$$
\begin{aligned}
& H_{u}-\left(H_{u^{\prime}}\right)^{\prime}+\left(H_{u^{\prime \prime}}\right)^{\prime \prime}=0, \\
& H_{r^{\prime}}-\left(H_{u^{\prime}}\right)^{\prime}+\left(H_{u^{\prime \prime}}\right)^{\prime \prime}=0,
\end{aligned}
$$

where now

$$
H=\kappa^{2}+\lambda(g-1),
$$

with the constraint $g=1$ given by Eqs. (8) and (9), and $\lambda(s)$ again a Lagrange function to be determined in the course of solving the system of differential equations. The latter consists of three equations, Eqs. (8), (18), and (19), for the three functions $u, v$, and $\lambda$. Equations (18) and (19) are fourth order in $u$ and $v$ and first order in $\lambda$, and so the system is of order nine. To get the system in normal form, Eq. (8) must be differentiated three times with respect to $s$. Reintegration of $g^{\prime \prime \prime}=0$ gives the respective fixed values 0 , 0 , and 1 to the resulting three constants of integration. The other six integration constants in the general solution are determined by the boundary conditions.

Because the side condition Eq. (8) does not involve $u^{\prime \prime}$ or $v^{\prime \prime}$, the boundary terms of the variation are not determined in the usual straightforward way. We nevertheless find the following six boundary terms if Eq. (8) can be solved for $v^{\prime}$ as a function of $u, v$, and $u^{\prime}$ to give $v^{\prime}=V\left(u, v, u^{\prime}\right)$ :

$$
\begin{gathered}
\left(H_{u^{\prime \prime}}+H_{l^{\prime \prime}}, V_{u^{\prime}}\right) \delta u^{\prime} \quad(s=0, l), \\
{\left[H_{u^{\prime}}-\left(H_{u^{\prime \prime}}\right)^{\prime}+H_{l^{\prime \prime}} V_{u}\right] \delta u \quad(s=0, l),} \\
{\left[H_{l^{\prime}}-\left(H_{l^{\prime \prime}}\right)^{\prime}+H_{v^{\prime \prime}} V_{l^{\prime}}\right] \delta v \quad(s=0, l) .}
\end{gathered}
$$

On the other hand, if $V_{u^{\prime}}$ is singular because it involves division by zero (as will happen in the subsequent development), then we must use a different set of six boundary terms, after solving Eq. (8) for $u^{\prime}=U\left(u, v, v^{\prime}\right)$,

$$
\begin{gathered}
\left(H_{l^{\prime \prime}}+H_{u^{\prime \prime}} U_{v^{\prime}}\right) \delta v^{\prime} \quad(s=0, l), \\
{\left[H_{v^{\prime}}-\left(H_{v^{\prime \prime}}\right)^{\prime}+H_{u^{\prime \prime}} U_{l^{\prime}}\right] \delta v \quad(s=0, l),} \\
{\left[H_{u^{\prime}}-\left(H_{u^{\prime \prime}}\right)^{\prime}+H_{u^{\prime \prime}} U_{u}\right] \delta u \quad(s=0, l) .}
\end{gathered}
$$

Suppose that we place no restrictions at all on the elastic line other than confinement to the surface. Then six integration constants are determined by setting equal to zero each of the six factors multiplying $\delta u, \delta v$, and $\delta u^{\prime}$ in the " $V$ boundary terms," or those multiplying $\delta u, \delta v$, and $\delta v^{\prime}$ in the " $U$ boundary terms." These boundary conditions are completely natural ( free) [8].

If natural conditions are allowed to prevail at $s=l$, but the initial position and direction of the elastic line are specified, then we get a different set of equations to determine the six integration constants. We illustrate with the $V$ boundary terms. Three boundary conditions are obtained by setting equal to zero each of the three factors 
multiplying $\delta u^{\prime}, \delta u$, and $\delta v$, with the factors evaluated at $s=l$. Two more boundary conditions are specified by the values of $u$ and $v$ at $s=0: u(0)=u_{0}, v(0)=v_{0}$. The unit tangent vector $\mathbf{t}(s)$ at any point $s$ of the curve is given by $\mathbf{x}_{u}(u, v) u^{\prime}(s)+\mathbf{x}_{v}(u, v) v^{\prime}(s)$, and so the initial tangent $\mathbf{t}(0)=\mathbf{x}_{u}\left(u_{0}, v_{0}\right) u^{\prime}(0)+\mathbf{x}_{r}\left(u_{0}, v_{0}\right) v^{\prime}(0)$. Specification of $u^{\prime}(0)$ $=u_{0}^{\prime}$ therefore completely determines the initial direction, since $v^{\prime}(0)$ is then fixed at the value $V\left(u_{0}, v_{0}, u_{0}^{\prime}\right)$. Thus, the required sixth boundary condition is that $u^{\prime}(0)$ have a specified value.

6. Elastic line on a plane. Let the origin of space be a point on the given plane $S$. Then $\mathbf{x}(u, v)=u \mathbf{i}+v \mathbf{j}$, where $(u, v)$ are rectangular coordinates, and $(\mathbf{i}, \mathbf{j})$ are unit vectors along the $u$ and $v$ axes, respectively. The vector $\mathbf{x}_{u}$ is $\mathbf{i}$, and $\mathbf{x}_{v}$, is $\mathbf{j}$. The coefficients of the first fundamental form are $E=G=1, F=0$. For the second fundamental form we find $L=M=N=0$. All the Christoffel symbols $\Gamma_{i j}^{k}$ also vanish.

It follows from Eq. (10) that the square normal curvature of any plane curve vanishes at all points of the curve. (This result is geometrically obvious; since the normal section of a plane at any point in any direction is a straight line.) In particular, any geodesic curve on the plane minimizes the total square normal curvature (giving to it the value zero), hence the total square curvature. The relaxed elastic line on a plane therefore lies along a geodesic.

The differential equations $\gamma_{1}=\gamma_{2}=0$ of geodesic curves become, for a plane, $u^{\prime \prime}=v^{\prime \prime}$ $=0$, the solutions of which are the straight lines $u=\alpha s+\gamma, v=\beta s+\delta, \alpha^{2}+\beta^{2}=1$. The elastic line relaxed on a plane assumes the form of a straight line.

7. Elastic line on a sphere. Let $S$ be a sphere of radius $R$. It is geometrically obvious, and can be verified from the general formulas, that $\kappa_{N}^{2}(s)$ is identically equal to $R^{-2}$ for any curve on $S$. The reason is that the normal section on a sphere at any point in any direction is a great circle. In particular, any geodesic on $S$ minimizes the total square normal curvature. Hence, an elastic line relaxed on a sphere lies along a geodesic. The differential equations for geodesic curves on a sphere have the great circles as solutions. Therefore a relaxed elastic line on a sphere has the trajectory of an arc of a great circle.

8. Elastic line on a cylinder. With the usual $x, y$, and $z$ axes of three-dimensional space, a vector $\mathbf{x}$ with components $(x, y, z)$ has its tip located on a circular cylinder of radius $R$ and vertical (long) axis the $z$ axis if

$$
\mathbf{x}=[R \cos (u / R), R \sin (u / R), v],
$$

where the surface coordinates $(u, v)$ are, respectively, length along the horizontal $(x-y$ plane) base circle measured in the counterclockwise sense when viewed from the positive $z$ axis, and length along a vertical generator. The ratio $u / R$ is just the ordinary polar angle of cylindrical coordinates; we choose $(u, v)$ to be lengths in orthogonal directions to keep track of units more easily and also to emphasize an analogy with the plane.

It can be checked that for these coordinates the first fundamental form for the cylinder and the plane are the same, $E=G=1, F=0$. It follows that, as for the plane, all the Christoffel symbols $\Gamma_{i j}^{k}$ are equal to zero. Consequently, the geodesics on a cylinder have 
the same equations as on a plane, $u(s)=\alpha s+\gamma, v(s)=\beta s+\delta, \alpha^{2}+\beta^{2}=1$. On a cylinder, these curves are the circular helices with radius $R$, pitch $2 \pi R(\beta / \alpha)$, and uniform curvature $R^{-1} \alpha^{2}$. The helix with infinite pitch, $\alpha=0, \beta= \pm 1$, is a vertical generator $u=\gamma=$ const; the helix with zero pitch has equations $u(s)=s+\gamma, v(s)=$ const, the representation of any of the horizontal circles on the cylinder.

The difference between a cylinder and a plane emerges when we consider the surface normal vectors. For the plane the surface normals are all parallel, but the normal vectors on a cylinder are parallel only along a generator; normals at points on different generators are distinct vectors. These facts are reflected in the corresponding second fundamental forms. The coefficients $L, M$, and $N$ all vanish for a plane; $M$ and $N$ equal zero on a cylinder, but $L=-R^{-1}$. Note that the second fundamental forms of the two surfaces become identical in the limit $R \rightarrow \infty$. From Eq. (10) the square normal curvature along any curve $[u(s), v(s)]$ on the cylinder is given by

$$
\kappa_{N}^{2}(s)=R^{-2} u^{\prime 4} .
$$

We can examine the incomplete variational problem. The expression for $H$ is

$$
H=R^{-2} u^{\prime 4}+\lambda\left(u^{\prime 2}+v^{\prime 2}\right)-\lambda,
$$

since the function $g$ in the side condition Eq. (8), given for the general surface by Eq. (9), becomes for a cylinder,

$$
g=u^{\prime 2}+v^{\prime 2}
$$

Note that $H$ contains neither $u$ nor $v$. The Euler equations (12) and (13) therefore have easy first integrals,

$$
H_{u^{\prime}}=\text { const }, \quad H_{u^{\prime}}=\text { const. }
$$

A cylinder is geometrically "homogeneous;" all points on it are equivalent. The only pertinent boundary conditions for the incomplete problem are the natural ones, Eq. (15). The integration constants in Eq. (31) must both be zero. Written out, the system of three differential equations for $u(s), v(s)$, and $\lambda(s)$ is

$$
\begin{aligned}
u^{\prime}\left(\lambda+2 R^{-2} u^{\prime 2}\right) & =0, \\
\lambda v^{\prime} & =0, \\
u^{\prime 2}+v^{\prime 2} & =1,
\end{aligned}
$$

and any solution of this system automatically satisfies the boundary conditions.

Any vertical generator $u(s)=$ const, $v(s)=s+$ const, along with $\lambda(s)=0$, gives a solution. From Eq. (28) the total square normal curvature is zero for a generator, and so this solution provides a minimum for $K_{N}$. A generator is a helix of infinite pitch, hence a geodesic. Therefore, an elastic line allowed to assume its minimum-energy trajectory on a cylinder adopts the form of a generator. The result that the path of a relaxed elastic line is along the only straight line possible on a cylinder is, of course, obvious even in the absence of any calculations.

There is another solution of the system of equations (32)-(34), the horizontal circle $u=s+$ const, $v=$ const. In this case, $\lambda(s)=-2 R^{-2}$. The square normal curvature at any point of the circle is $R^{-2}$ from Eq. (28), so this curve does not give a minimum. 
Analysis of the complete variational problem leads to more interesting results. We calculate the square curvature of any curve on a cylinder from Eqs. (18)-(21) and Eq. (28),

$$
\kappa^{2}=u^{\prime \prime 2}+v^{\prime \prime 2}+R^{-2} u^{\prime 4} .
$$

The function $g$ is the same, given by Eq. (30), and so again $H$, now equal to $\kappa^{2}+\lambda(g-1)$, does not depend on $u$ or $v, H_{u}=H_{v}=0$. First integrals of the Euler equations, Eqs. (22) and (23), are thus

$$
\begin{aligned}
& H_{u^{\prime}}-\left(H_{u^{\prime \prime}}\right)^{\prime}=\text { const }, \\
& H_{v^{\prime}}-\left(H_{v^{\prime \prime}}\right)^{\prime}=\text { const. }
\end{aligned}
$$

We impose natural boundary conditions at $s=l$, so that the factors multiplying $\delta u^{\prime}$, $\delta u$, and $\delta v$ in the set of boundary terms (25) are equal to zero when evaluated at $s=l$. From Eqs. (8) and (30), the function $V^{2}$ equals $1-u^{\prime 2}$, and so $V_{u}=V_{v}=0$. Comparison of the last two boundary terms of the set (25) with the left-hand sides of Eqs. (36) and (37) shows that both integration constants in the latter equations are equal to zero. The same conclusion is reached by consideration of the set (26) of " $U$ boundary terms." When written out, the Euler equations (36) and (37) become

$$
\begin{gathered}
u^{\prime \prime \prime}-u^{\prime}\left(\lambda+2 R^{-2} u^{\prime 2}\right)=0, \\
v^{\prime \prime \prime}-\lambda v^{\prime}=0 .
\end{gathered}
$$

Together with Eq. (34), which expresses the constraint $g=1$, we have a system of three equations for $u(s), v(s)$, and $\lambda(s)$.

We have already "used up" two boundary conditions at $s=l$ in the determination of the integration constants in Eqs. (36) and (37). If the $V$ boundary terms are used, then the factor multiplying $\delta u^{\prime}$ in set (25) equals zero at $s=l$,

$$
u^{\prime \prime} \mp u^{\prime} v^{\prime \prime}\left(1-u^{\prime 2}\right)^{-1 / 2}=0 \quad(s=l) \text {, }
$$

where the upper (lower) sign applies if $V$ is chosen as the positive (negative) root of $1-u^{\prime 2}$. If $V_{u^{\prime}}$ does not exist at $s=l$, that is, if $u^{\prime 2}(l)=1$, then we must use the $U$ boundary terms. Equation (40) is replaced by

$$
v^{\prime \prime} \mp v^{\prime} u^{\prime \prime}\left(1-v^{\prime 2}\right)^{-1 / 2}=0 \quad(s=l),
$$

where the upper (lower) sign applies if $U$ is chosen as the positive (negative) root of $1-v^{\prime 2}$.

The boundary conditions are completed by specification of the initial point and direction,

$$
u(0)=0, \quad v(0)=0, \quad u^{\prime}(0)=\alpha .
$$

From Eq. (27) and the first two initial conditions, we see that the elastic line starts at a point on the $x$ axis at distance $R$ from the origin. From the discussion of the tangent vector in the section on the complete variational problem, we calculate that the initial unit tangent vector $\mathbf{t}_{0}$, as determined by Eq. (42), is $\alpha \mathbf{j} \pm \sqrt{1-\alpha^{2}} \mathbf{k}$, where $(\mathbf{j}, \mathbf{k})$ are the unit vectors along the $y$ and $z$ axes. 
Suppose that the initial direction is along the vertical, that is, $\alpha=0, \mathbf{t}_{0}= \pm \mathbf{k}$. Then we can check that the generator $u(s)=0, v(s)= \pm s$ is a solution, together with $\lambda(s)=0$; equations (34), (38), and (39) are satisfied, as are the boundary conditions (40) and (42). As we already know, an elastic line initially directed along a generator follows the generator along its whole length when relaxed.

Let the initial direction be horizontal, $\alpha^{2}=1, \mathbf{t}= \pm \mathbf{j}$. The horizontal circle $u(s)= \pm s$, $v(s)=0$ is a solution, coupled with $\lambda(s)=-2 R^{-2}$. The system of differential equations (34), (38), and (39) is satisfied, and so are the boundary conditions (41) and (42). This case is deceptive, however; we will show in a subsequeni paper that a horizontal circular arc for an elastic line constrained at $s=0$ to the horizontal direction becomes an unstable equilibrium trajectory when the length of the line exceeds a certain critical value.

Thus far, the equilibrium trajectories are geodesics, the helices of infinite and zero pitch, respectively. Let now $0<|\alpha|<1$, so that the initial direction is oblique to a generator. It is reasonable (if we don't think about it too much) to expect that the relaxed elastic line will conform to the unique helix with the specified initial direction, $u(s)=\alpha s, v(s)=$ $\pm \sqrt{1-\alpha^{2}} s$. [We have to say whether $v(s)$ proceeds up (plus) or down (minus). The corresponding helix is either right-handed or left-handed, respectively, if $\alpha$ is chosen to be positive.] This trial solution satisfies the boundary equation (40), or, equivalently, (41). The boundary equation (42) is also obeyed, as is the differential equation (34). To satisfy the Euler equation (39) we must choose $\lambda(s)=0$. But now we see that the helix does not satisfy the Euler equation (38). The relaxed elastic line, forced to start in a given oblique direction, does not wind around the cylinder along the corresponding helix.

9. Elastic line on a pseudotorus. The vector components $(x, y, z)$ of a point on a torus centered at the origin are

$$
\begin{aligned}
& x=a\left(1+\frac{b}{a} \cos \frac{u}{b}\right) \cos \frac{v}{a}, \\
& y=a\left(1+\frac{b}{a} \cos \frac{u}{b}\right) \sin \frac{v}{a}, \\
& z=b \sin \frac{u}{b},
\end{aligned}
$$

where $b$ is the radius of the transverse circular cross section, and $a$ is the distance from the origin to the center of the cross section (i.e., $a+b$ is the outer radius of the torus, $a-b$ the inner radius). Circumferential length around the cross section of radius $b$ is given by $u$, with $v$ the length along the central circle of radius $a$. It can be checked that the components of the first and second fundamental forms are, respectively,

$$
E=1, \quad F=0, \quad G=\left(1+\frac{b}{a} \cos \frac{u}{b}\right)^{2},
$$

and

$$
L=b^{-1}, \quad M=0, \quad N=a^{-1}\left(1+\frac{b}{a} \cos \frac{u}{b}\right) \cos \frac{u}{b}
$$


A pseudotorus may be defined by the replacement of $1+(b / a) \cos (u / b)$ with unity in the components of the first and second fundamental forms:

$$
\begin{gathered}
\quad E=1, \quad F=0, \quad G=1, \\
' L=b^{-1}, \quad M=0, \quad N=a^{-1} \cos (u / b) .
\end{gathered}
$$

From Eqs. (43)-(45) it is seen that a pseudotorus may be visualized as a very thin torus (very narrow cross section). Comparison with the fundamental forms of a cylinder shows that the first forms are identical, while the essential difference in the second forms resides in the nonvanishing value of $N$ for the pseudotorus, which reflects the additional circumferential variation in orientation of the surface normal along the $v$-coordinate.

A torus may be constructed from a cylinder with ends by bending the cylinder until the ends merge. In this process the inner generators are contracted while the outer ones must stretch. The stretching and contraction imply that the first fundamental forms of the cylinder and torus are different. By contrast, the cylinder and pseudotorus have the same metric (first fundamental form). The pseudotorus is obtained from a cylinder with ends by the imaginary process of bending the cylinder while leaving the lengths of all generators invariant.

The major advantage gained by consideration of the pseudotorus is that its geodesics have the same equations as for the plane and cylinder, $u(s)=\alpha s+\gamma, v(s)=\beta s+\delta$, $\alpha^{2}+\beta^{2}=1$. These curves wind around the torus in a manner that may be visualized from the helices on a cylinder when the cylinder is bent to form a torus. Among the geodesics on a pseudotorus are the outer ring $u=0, v=s+\delta$; the inner ring $u=\pi b$, $v=s+\delta$; and indeed any circle $u=$ const. The transverse circles $v=$ const are also geodesics.

The circles $u=$ const and $v=$ const are examples of closed geodesics. Most geodesics on a pseudotorus are not closed. For a geodesic to be closed, it must complete an integral number $n$ of turns along the $v$-coordinate when the number $m$ of turns along the $u$-coordinate is integral. From the equations for any geodesic we find the characteristic of a closed geodesic, $m / n= \pm(a / b)\left(\alpha / \sqrt{1-\alpha^{2}}\right)$; that is, $(a / b)\left(\alpha / \sqrt{1-\alpha^{2}}\right)$ must be a rational number. Since $0 \leqslant \alpha / \sqrt{1-\alpha^{2}}<\infty$, the quantity $\pm(a / b)\left(\alpha / \sqrt{1-\alpha^{2}}\right)$ sweeps through all real numbers as $\alpha$ sweeps from zero to unity. When a rational number is hit, the corresponding geodesic is closed, but this circumstance "almost never" happens. Nearly all geodesics wind endlessly around the torus without closing.

The Gaussian curvature of the pseudotorus is given by $(a b)^{-1} \cos (u / b)$ and is therefore negative on the inner part of the pseudotorus $\pi / 2<u / b<3 \pi / 2$. A surface of negative Gaussian curvature is saddle-shaped in the neighborhood of any point. A family of curves called asymptotic may be defined in regions of negative curvature but not in regions of positive curvature [7]. Thus, asymptotic curves do not exist on cylinders or spheres, or on the outer part of a torus or pseudotorus. But a family of asymptotic curves does exist on the inner part of a torus or pseudotorus. It is a characteristic of asymptotic curves that the normal curvature $\kappa_{N}$ vanishes identically along them. Therefore, the asymptotic curves solve the incomplete variational problem. But the asymptotic curves are not geodesics, so the incomplete variational problem cannot provide information about relaxed elastic lines on a torus or pseudotorus. 
To analyze the complete variational problem for the pseudotorus we construct the function $H=\kappa_{g}^{2}+\kappa_{N}^{2}+\lambda(g-1)$, where $\kappa_{g}^{2}$, given generally by Eq. (19), is the same as for the cylinder, $\kappa_{g}^{2}=u^{\prime \prime 2}+v^{\prime \prime 2}$, but $\kappa_{N}^{2}$, Eq. (10), is more complicated, $\kappa_{N}^{2}=b^{-2}\left[u^{\prime 2}+\right.$ $\left.(b / a) v^{\prime 2} \cos (u / b)\right]^{2}$. The constraint function $g$ is the same as for the cylinder, Eq. (30). The following conclusions may be verified by straightforward but tedious checking.

(1) The outer ring $u=0$ minimizes the elastic energy if natural boundary conditions are used both at $s=0$ and at $s=l$. An elastic line free to wander on the surface of a pseudotorus will preferentially lie along a geodesic, the outer ring. Other geodesic solutions of this problem (i.e., equilibrium paths) are the transverse cross sections $v=$ const, the inner ring $u=\pi b$, and the top and bottom rings $u=\frac{1}{2} \pi b, u=\frac{3}{2} \pi b$, but these geodesics do not have minimum curvature. The other circles $u=\gamma\left[\gamma \neq 0, \frac{1}{2} \pi b, \pi b\right.$, or $\left.\frac{3}{2} \pi b\right]$, are geodesics but not equilibrium paths for the elastic line.

(2) If an initial point $(u, v)=(\gamma, \delta)$ and direction $\left(u^{\prime}, v^{\prime}\right)=\left(\alpha, \pm \sqrt{1-\alpha^{2}}\right)$ are specified, the relaxed elastic line does not lie along the corresponding geodesic $u=\alpha s+\gamma$, $v= \pm \sqrt{1-\alpha^{2}} s+\delta$ for $0<\alpha<1$, that is, if the initial direction is oblique.

(3) If $\alpha=1$, that is, if the initial direction is along a transverse circle $v=$ const, then the corresponding geodesic (the transverse circle) is an equilibrium path for the elastic line, but we expect it to be unstable for lengths exceeding a critical value.

(4) If $\alpha=0, \gamma=0, \frac{1}{2} \pi b, \pi b$, or $\frac{3}{2} \pi b$, the corresponding geodesics are equilibrium paths (outer, top, inner, and bottom circles, respectively). Our expectation is that only the outer ring is a stable trajectory for an elastic line of any length.

(5) If $\alpha=0, \gamma \neq 0, \frac{1}{2} \pi b, \pi b$, or $\frac{3}{2} \pi b$, the corresponding geodesics $u=\gamma$ are not equilibrium trajectories for the elastic line.

\section{REFERENCES}

[1] D. Hilbert and S. Cohn-Vossen, Geometry and the imagination, Chelsea, New York, 1952

[2] L. D. Landau and E. M. Lifshitz, Theory of elasticity, Pergamon Press, Oxford, 1979, p. 84

[3] J. D. McGhee and G. Felsenfeld, Nucleosome structure, Ann. Rev. Biochem. 49, 1115-1156 (1980)

[4] T. J. Richmond, J. T. Finch, B. Rushton, D. Rhodes, and A. Klug, Structure of the nucleosome core particle at 7 A resolution. Nature 311, 532-537 (1984)

[5] J. D. McGhee, D. C. Rau, E. Charney, and G. Felsenfeld, Orientation of the nucleosome within the higher order structure of chromatin. Cell 22, 87-96 (1980)

[6] J. Widom and R. L. Baldwin, Monomolecular condensation of DNA induced by cobalt hexamine, Biopolymers 22. $1595-1620(1983)$

[7] C.-C. Hsiung, A first course in differential geometry, John Wiley \& Sons, New York, 1981

[8] R. Weinstock, Calculus of variations, Dover, New York, 1974 